\title{
Moesin expression is correlated with its involvement in patients with Behcet's disease
}

\author{
Muhammad Hussain ${ }^{1}$, Peng Chen ${ }^{1,2}$, Yixuan Zhang ${ }^{1}$, Yaping Tian ${ }^{3}$, Hongwu Du ${ }^{1}$
}

\author{
${ }^{1} 112$ Lab, School of Chemistry and Biological Engineering, University of Science and \\ Technology, Beijing, China \\ 2Experimental Research Center, China Academy of Traditional Chinese Medicine, \\ Beijing, China \\ ${ }^{3}$ Department of Clinical Biochemistry, Chinese PLA General Hospital, Beijing, China
}

Submitted: 6 March 2018

Accepted: 16 August 2018

Arch Med Sci 2020; 16 (4): 924-930

DOI: https://doi.org/10.5114/aoms.2020.92911

Copyright $\odot 2020$ Termedia \& Banach

\section{Abstract}

Introduction: Behcet's disease (BD) is a rare, chronic autoimmune disorder of unknown etiology. Although the profile of autoantibodies for this disease is not yet completely understood, because of better disease recognition, its prevalence is increasing throughout the world. Among ERM proteins (ez$\mathrm{rin} / \mathrm{radixin/moesin),} \mathrm{moesin} \mathrm{is} \mathrm{a} \mathrm{member} \mathrm{of} \mathrm{a} \mathrm{family} \mathrm{which} \mathrm{is} \mathrm{involved} \mathrm{in}$ autoimmune diseases. The aim of this study is to confirm whether moesin is a potential anti-endothelial cell autoantigen (AECA) in Hans Chinese BD patients.

Material and methods: First, a full length recombinant human moesin protein was over-expressed and purified. Second, it was identified by mass spectrometry and then purified moesin was used to perform Western blotting, immunoprecipitation and ELISA with confirmed BD patients. Finally, in vitro cytotoxicity experiments were conducted with anti-moesin antibodies by the resazurin reduction assay method.

Results: Purified moesin protein was successfully expressed and then its antigenicity was confirmed by Western blotting and immunoprecipitation techniques. Anti-moesin antibodies were detected in approximately onethird (38\%) of BD patients by ELISA and the reactivity of BD serum IgG antibodies against moesin was found to be significantly higher than HC ( $p<$ 0.0001 ). Moreover, in order to validate our results, cytotoxicity experiments also confirmed that anti-moesin antibody had a significant inhibitory effect on endothelial cell activity.

Conclusions: Expression is correlated with the involvement of moesin as an autoantigen in BD pathology, which is a new finding. It might be a new candidate biomarker in the Han Chinese population.

Key words: EAhy926, autoantigen, moesin, endothelial cells.

\section{Introduction}

Behçet's disease (BD) is characterized by a complex of triple symptoms: genital, oral ulcer and ophthalmitis [1]. Over the past 20 years, although researchers have made great progress, the etiology of this disease is still unknown and the corresponding laboratory diagnosis is still needed. Endothelial cells are activated by anti-endothelial cell antibodies (AECA) and inflammatory responses have been found to play a vital role in autoimmune pathological processes [2]. AECA have been detected in

\author{
Corresponding author: \\ Prof. Dr. Hongwu Du \\ School of Chemistry and \\ Biological Engineering \\ University of Science \\ and Technology \\ 30 Xueyuan Road \\ Haidian District \\ Beijing 100083, China \\ Phone: +861062334497 \\ Fax: +861062332462. \\ E-mail: hwdu93@126.com
}


BD patients and proven to be associated with vasculitis symptoms $[3,4]$. In the past decades, most studies focused on the pathogenesis of AECA and findings of potential biomarkers to explain the etiology of BD. Recently, at least four new targets of AECA in BD, i.e. prohibitin, HSP27, ETFB and annexin $A 2$, have been successfully screened out by our group [5-9]. These findings not only added knowledge to explain the AECA related pathology, but also further confirmed the key role of AECA in the process of $\mathrm{BD}$.

ERM proteins (ezrin/radixin/moesin) are considered as cross linkers as well as signal transducers between plasma membrane and actin-cytoskeleton. Moesin is a member of the ERM family which acts as a membrane organizing extensions, involved in the formation of microvilli. Moesin is also an important member of structural proteins in the cellular membranes [10]. Studies revealed that moesin plays a key role in the development and exacerbation of vasculitis as a potential AECA autoantigen and the endothelial cells can be activated by anti-moesin antibodies [11, 12]. Correlation of moesin and AECA has been proved already, and it could mediate human pulmonary microvascular endothelial cell damage, which is depicted in early apoptosis and dysfunction of endothelial skeletal proteins [13]. Moesin specific antibodies were also frequently detected in patients with acquired aplastic anemia, which have a close relationship with various cytokines produced by $C D 4^{+} T$ cells [14]. At least two important inflammatory cytokines (TNF- $\alpha$, IFN- $\gamma$ ) in patients having peripheral blood mononuclear cells with BD have been detected at an unusually high level, which were stimulated by anti-moesin antibodies $[15,16]$.

The goal of our present study is to evaluate whether moesin is an AECA autoantigen involved in pathogenesis of BD. To test our hypothesis, Western blotting, immunoprecipitation, cytotoxicity and in house ELISA were developed and validated clinically through screening of a set of newly diagnosed patients.

\section{Material and methods}

\section{Subjects}

One hundred and fifty subjects were enrolled in this study and evaluated by serological methods. The recurrent data from involved organs such as oral ulcer, uveitis and genital ulcer were recorded. Serum samples were categorized into three groups: (1) experimental group (BD) $(n=50)$ with an average age of 37 (25 males, 25 females); (2) a rheumatoid arthritis (RA) disease control group (as studies revealed that moesin sera from patients with RA had anti-ERM antibodies and moesin acts as an antigen in rheumatoid diseases, RA was chosen) ( $n=50)$ with an average age of 51 (9 males, 41 females); (3) a healthy control group $(\mathrm{HC})(n=50)$ with an average age of 23 (14 males, 36 females). Diagnosed BD patients fulfilled the criteria as defined by an international group [17]. Serum samples from all three groups (BD patients, RA and healthy controls) were collected from Chinese PLA General Hospital (Beijing). Ethical approval for this study was taken from the Chinese PLA General Hospital ethical committee and consent was taken from each patient. All serum samples were aliquoted and stored at $-70^{\circ} \mathrm{C}$ for further use.

\section{Moesin cloning, expression and purification}

This method was basically performed as described before [18]. Extraction of RNA from EA.hy926 cells (human endothelial cell line) was carried out as per TRIzol reagent kit instructions (Invitrogen, CA). Then gene amplification was performed by RT-PCR using kit instructions (Fermentas, MD). Primer sequences were taken as:

5'-CCTGAATTCCCCAAAACGATCAGTGTG-3 (Forward),

5' -CCCAAGCTTTTATTAGCGCAGGGTCTTG TATTT-3' (Reverse).

The PCR product of the moesin gene was sequenced by Sangon Biology (Shanghai, China). Human moesin protein was over-expressed in E. coli bacterium BL21 and then the recombinant moesin was purified by the Ni-NTA kit (Qiagen, Hilden, Germany). Protein concentration was measured using a BCA kit (Biosynthesis Biotechnology, Beijing, China). Purified recombinant protein was confirmed by proteomics analyzer $A B 4700$ mass spectrometry (Applied Biosystems, Foster City, CA).

\section{In-gel digestion and mass spectrometry analysis}

In-gel digestion and mass spectrometry were performed as described previously [19]. The excised gel pieces were destained with $50 \%$ acetonitrile plus $25 \mathrm{mM} \mathrm{NH}_{4} \mathrm{HCO}_{3}$ and dried by vacuum centrifugation, followed by addition of $25 \mathrm{mM}$ $\mathrm{NH}_{4} \mathrm{HCO}_{3}$ in $10 \mathrm{mM}$ dithiothreitol to reduce it for $1 \mathrm{~h}$ at $57^{\circ} \mathrm{C}$. Then DTT solution was replaced by $55 \mathrm{mM}$ iodoacetamide and $25 \mathrm{mM} \mathrm{NH}_{4} \mathrm{HCO}_{3}$ and left for incubation at room temperature for $45 \mathrm{~min}$ in the dark. The liquid phase was dehydrated and the gel was dried in a vacuum concentrator. Then the gel was covered with $30 \mu \mathrm{l}$ of the $0.05 \mathrm{mM}$ $\mathrm{NH}_{4} \mathrm{HCO}_{3}$ trypsin containing buffer (Sigma, MO), and overnight digestion was performed at $37^{\circ} \mathrm{C}$. Analysis of mass spectrum data was performed with the search engine Mascot database (Matrix Sciences, London, UK). 


\section{Western blotting}

$12 \%$ polyacrylamide gel was prepared and a $20 \mu \mathrm{l}$ sample (loading buffer + recombinant moesin) was loaded into the well to separate and identify the recombinant human moesin. Human IgG protein was also loaded as a positive control $(20 \mu \mathrm{l})$. The gel was relocated on polyvinylidene fluoride membranes (PVDF; Merck Millipore, MA). Skim milk (5\%) was used to block the PVDF membranes. Then $5 \mathrm{BD}$ and $5 \mathrm{HC}$ sera were randomly selected (1: 500 dilution) and incubated at $4^{\circ} \mathrm{C}$ for $12 \mathrm{~h}$. The unbound antibodies were removed after washing membranes 5 times with $1 \%$ PBST (phosphate buffered saline Tween-20). Secondary antibody, i.e. HRP/lgG goat anti-human (1 : 2000 dilution) (ImmunoHunt, Beijing, China) was added and incubated for $60 \mathrm{~min}$ at $37^{\circ} \mathrm{C}$. Finally, an $E C L$ mix was prepared (Solution A \& B) and the membranes were incubated according to the kit instructions (Applygen, Beijing, China)

\section{Immunoprecipitation}

Recombinant moesin protein $(3 \mu \mathrm{g})$ was mixed with $2 \mathrm{BD}$ sera and incubated at temperature $4^{\circ} \mathrm{C}$ overnight. The beads of Sepharose A were washed in PBS and put on the plate on a shaker at $4^{\circ} \mathrm{C}$ for $2 \mathrm{~h}$. Then samples were centrifuged for $5 \mathrm{~min}$ at $3000 \mathrm{rpm}$ to obtain the immunoprecipitates. Then $0.5 \%$ PBST, $500 \mu \mathrm{l}$, was used to wash the immunoprecipitates three times. Then both immunoprecipitates and the supernatant (after the first centrifuge, before washing three times) were mixed with loading buffer and determined by SDS-PAGE. Coomassie blue (Sigma, MO) was used to stain the gels in both experiments.

\section{ELISA}

Recombinant moesin $(100 \mathrm{ng} / \mathrm{ml})$ was added to 96 microplate wells (Corning, NY) and the plates were kept at $4^{\circ} \mathrm{C}$ overnight. The plates were washed 3 times with PBST and blocked with $200 \mu \mathrm{l}$ ( $5 \%$ goat serum), then incubated for $2 \mathrm{~h}$ at $37^{\circ} \mathrm{C}$. The plates were washed three times and $100 \mu \mathrm{l}$ of sera (diluted 1 : 100 in PBS) was added and incubated for $2 \mathrm{~h}$ at $37^{\circ} \mathrm{C}$. The plates were washed again and $100 \mu \mathrm{l}$ of secondary antibody lgG/HRP (goat anti-humans) (ImmunoHunt, Beijing, China) was added and plates were incubated at $37^{\circ} \mathrm{C}$ for 1 h. Optical density was measured at 450 and $620 \mathrm{~nm}$ with a plate reader (Tecan, Hombrechtikon, Switzerland). The ELISA method was also used to test the binding ability of anti-moesin antibody. The same procedure was used, except the first antibody was anti-moesin antibody (Bioss, Beijing, China) diluted 1: 100 in PBS for $30 \mathrm{~min}$ at $37^{\circ} \mathrm{C}$, and the secondary antibody was HRP labeled goat anti-mouse IgG.

\section{Resazurin assay}

The resazurin assay plate method was described in previous studies [20]. EA.hy926 cells were coated on plates (Coring, NY) and left to grow overnight (cell growth observed was $80 \%$ ). Next, wells with cells were divided into two groups. In group one, we added $5 \mu \mathrm{l}$ of resazurin in $50 \mu \mathrm{l}$ of DMEM (Dulbecco's Modified Eagle Medium) with anti-moesin antibodies (Bioss, Beijing, China), while in the other group $50 \mu \mathrm{l}$ of DMEM medium was added as a blank control and then $10 \mu \mathrm{l}$ of resazurin solution was added to each well. Color change from blue to pink indicated growth of cells and the absorbance was measured with a plate reader (Tecan, Hombrechtikon, Switzerland) at $620 \mathrm{~nm}$ with different time intervals $(0,12,18$, $30 \mathrm{~h}$ ) after the addition of resazurin. The statistical analysis for resazurin assay was carried out by the $t$-test method.

\section{Statistical analysis}

SPSS software (Version 17, IL) was used to perform the $t$-test. $P$-value $<0.05$ was taken as significant. The positive definition for the critical point was considered as a higher value than the healthy control (mean + 2 SD).

\section{Results}

\section{Moesin cloning, expression and purification}

RT-PCR technique was used to amplify the target gene (human moesin), which is depicted after gel electrophoresis (Figure 1 A). A 75 kDa protein band was seen, which was confirmed as a moesin protein band when compared with E. coli BL21 (DE3) recombinant strain without IPTG induction. His-tag moesin was obtained by a resin Ni-NTA kit (Figure $1 \mathrm{~B}$ ). The protein obtained after resin purification was confirmed by mass spectrometry. Protein score $>70$ is significant and a $p$-value $<0.05$ is to hit a protein rank as shown in Figure $1 \mathrm{C}$.

\section{Western blotting and immunoprecipitation}

Recombinant moesin associated sera of $5 \mathrm{BD}$, $5 \mathrm{RA}$ and $5 \mathrm{HC}$ were randomly selected from the repository of sera bank. 2 BD sera out of $5 \mathrm{BD}$ patients demonstrated antigen-antibody positive reaction and all 5 healthy people's sera showed no reaction, indicating that moesin is a probable BD patient's autoantigen (Figure 2 A). Similarly, immunoprecipitation results of BD1 and BD2 samples were consistent and matched with the Western blotting results (Figure $2 \mathrm{~B}$ ).

\section{ELISA}

Enzyme linked immunosorbent assay (ELISA) of recombinant moesin was carried out with 
A

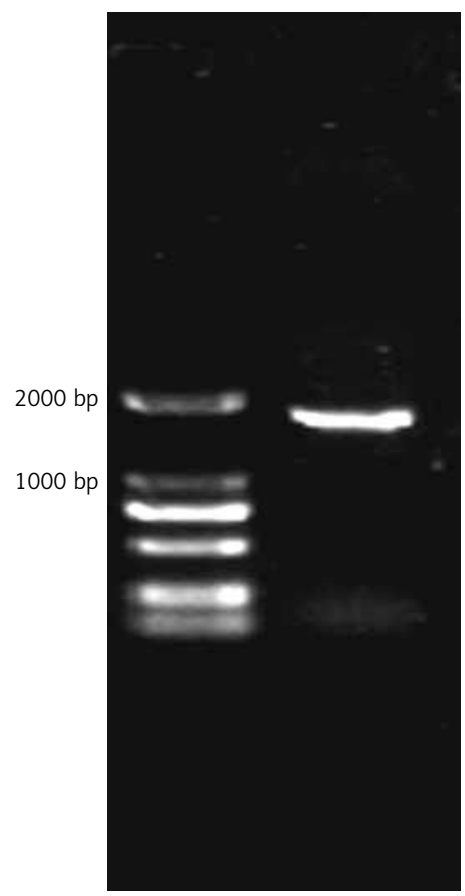

Marker DNA

C

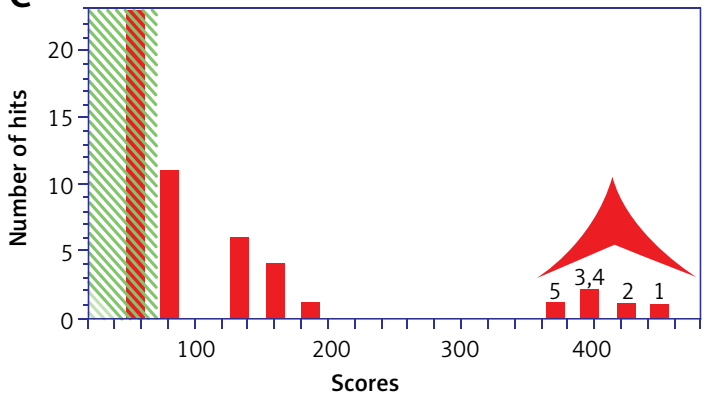

B

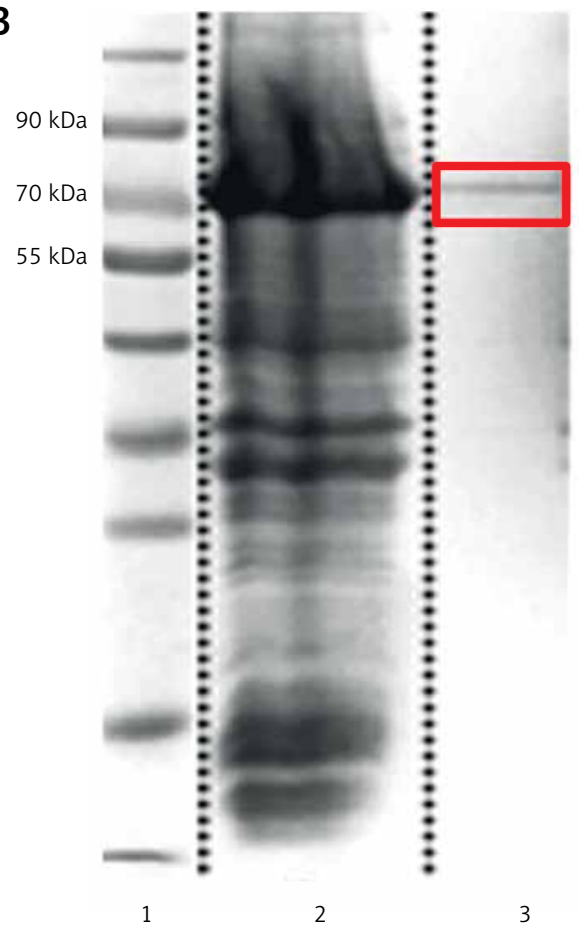

\begin{tabular}{|l|c|c|}
\hline Accession & Mass & Score \\
\hline MOES human & 67892 & 450 \\
\hline MOES mouse & 67839 & 419 \\
\hline MOES bovin & 68047 & 407 \\
\hline MOES rat & 67868 & 403 \\
\hline MOES pig & 67733 & 375 \\
\hline
\end{tabular}

Figure 1. Moesin gene amplification, identification and verification. A - Human moesin gene was amplified by polymerase chain reaction technique. Left band is DNA weight marker, right band is target gene $\sim 2000 \mathrm{bp}$. B - The human moesin protein was expressed and purified. Lane 1: protein marker, Lane 2: human moesin protein was over-expressed in E. coli BL21 after IPTG-induction, Lane 3: the moesin protein was purified using Ni-NTA resin. C - Verification of purified moesin protein by mass spectrometry; i.e. moesin protein score obtained is 450 while protein score $>70$ is significant $(p<0.05)$

50 BD patients' sera to confirm the pathology in BD. At the same time, 50 RA and 50 healthy people's sera were taken for ELISA. Results were noted and anti-lgG antibody reaction against recombinant moesin was observed in 19 out of 50 patients' BD sera (38\%), 12 out of 50 RA patients (24\%), and 2 out of $50 \mathrm{HC}(4 \%)$. The reactivity of BD serum IgG antibodies against moesin was significantly higher than RA $(p=0.0432)$ and $\mathrm{HC}(p<0.0001)$ (Figure $3 \mathrm{~A})$.

\section{Clinical significance of BD patients}

Moesin's relationship with clinical symptoms in BD patients was analyzed. Nineteen out of 50 BD subjects showed a positive reaction with the recombinant human moesin protein. On the other hand, clinical information with anti-moesin was as follows: 19 antibody-positive and 31 antibody-negative subjects were found; no significant differences were found in clinical symptoms. All clinical information regarding BD patients are summarized in Figure 3 B.

\section{Resazurin assay experiments}

Moesin polyclonal antibody was tested by ELISA. Target moesin protein could be strong and can be recognized by polyclonal antibody (Figure $3 \mathrm{C}$ ). This method confirmed that the polyclonal antibody of moesin can bind to moesin protein. The absorbance difference of each group at $620 \mathrm{~nm}$ was shown in Figure $3 \mathrm{D}$. The negative group showed a stronger reducing activity than the experimental group with commercial antimoesin antibody $(p<0.001)$, indicating that anti- 
A

BD

Moesin

IgG H-chain

RA

IgG H-chain

$\mathrm{HC}$

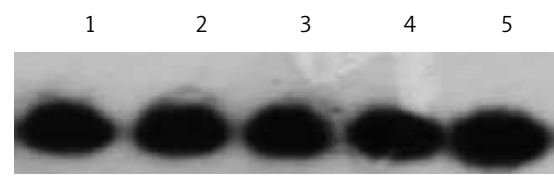

B

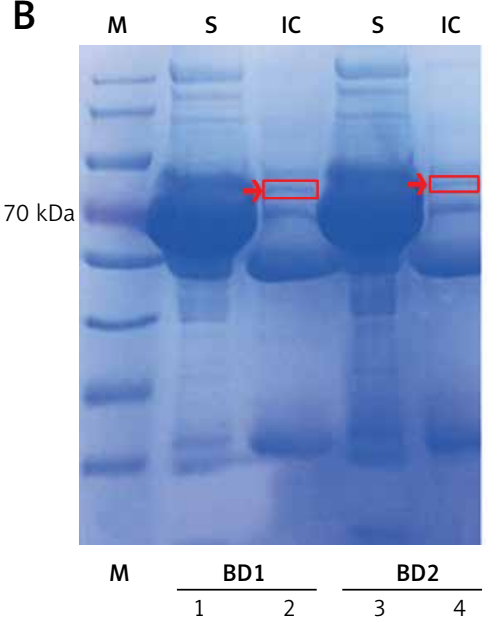

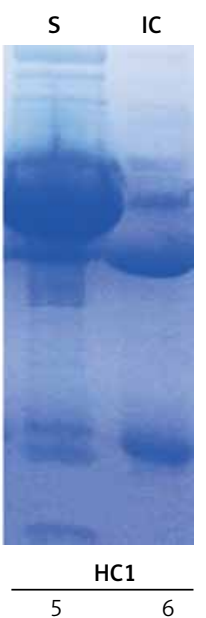

Figure 2. Demonstration of moesin antigenicity in BD. A - Western blotting results: two positive results among the $5 \mathrm{BD}$ patients were BD1 and BD2. About $75 \mathrm{kDa}$ band was observed. All healthy controls showed negative results. Human IgG protein was taken as positive control. B - Immunoprecipitation results: two positive results (BD1 and BD2) were then used to perform immunoprecipitation to further confirm the antigenicity of moesin. The moesin protein band clearly existed in immune complex (IC) (antigen-lgG complexes, lane 2, 4 in red boxes) by mixing BD1 and BD2 patients' sera. HC1 sample was performed as a control here; no target band was detected in immune complex in lane 6. Lane 1, 3, 5: the supernatant (S) of immunoprecipitation and also no band of moesin was observed

A

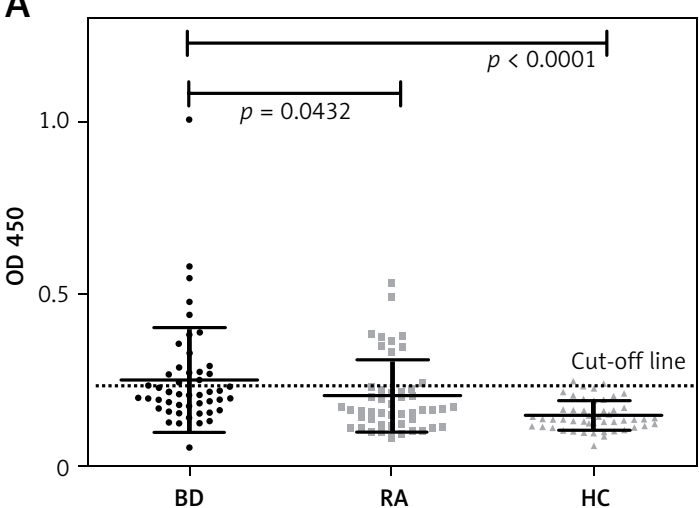

C

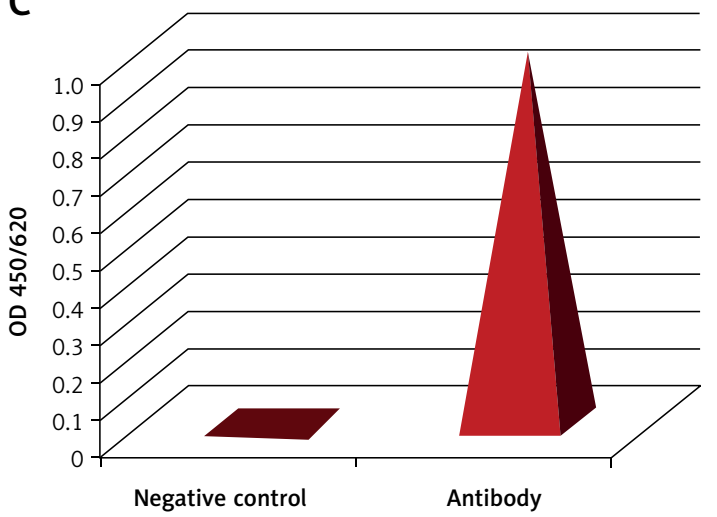

B

GI
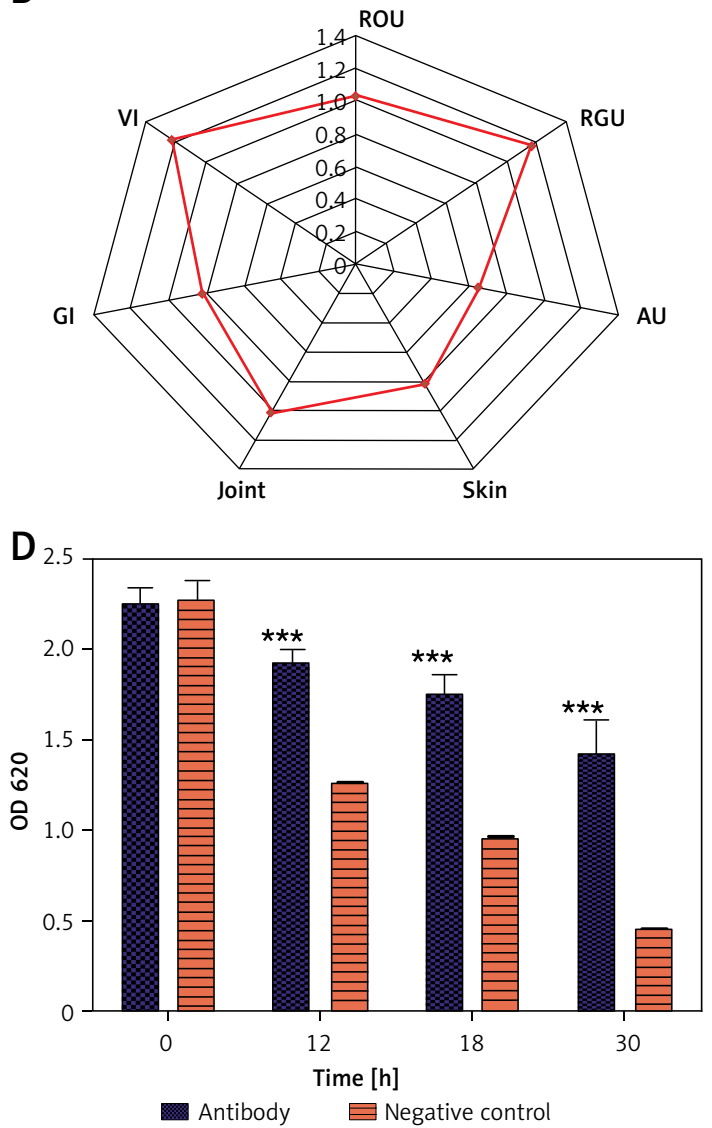

Figure 3. ELISA, clinical information analysis and cytotoxicity experiments. A - The reactivity of BD sera IgG antibodies against recombinant human moesin protein was detected by ELISA method. Data, analyzed by $t$ test, were expressed as mean \pm SD. B - Clinical information comparison between anti-moesin antibody-positive and negative groups. The ratio was obtained by calculating (number clinical+/number positive)/(number clinical+/number negative). C - Right peak: target moesin protein could be strongly recognized by purified polyclonal antibody. Left peak is the blank control. D - Resazurin assay results: the absorbance differences of each group at $620 \mathrm{~nm}$ are shown. ${ }^{* *} P<0.001$

$R O U$ - recurrent oral ulcers, RGU - recurrent genital ulcers, SKIN - skin lesions, AU - anterior uveitis, VI - vascular involvement, GI gastrointestinal involvement, JOINT - joint involvement. Antibody - experiment group with anti-moesin antibody sera, Ctrl - control. 
moesin antibody might inhibit the activity of endothelial cells.

\section{Discussion}

Autoimmune diseases arise from an abnormal response of the immune system against our own cells and tissues. Many autoimmune diseases are categorized on the basis of undesirable activity of autoantibodies. These antibodies recognize and bind to often normal and healthy autoantigens, thereby causing substantial damage and failure of relevant tissues and organs.

In this study we found autoantibodies in BD patients with one such target antigen, moesin. To achieve this, we developed an ELISA method which is highly sensitive and used for the detection of serum autoantibodies. Studies revealed that ELISA is more reliable and an established method for detecting anti-ERM antibodies [21]. In our results, anti-moesin antibodies were observed in 19 out of 50 patients' BD sera (38\%), 12 out of 50 RA patients (24\%), 2 out of $50 \mathrm{HC}(4 \%)$ and the reactivity of BD serum against moesin was significantly higher than RA and HC. In a previous study approximately $30 \%$ of the sera from patients with RA had anti-ERM antibodies and moesin acted as an antigen in rheumatoid diseases [22]. Therefore, we chosen RA as a disease control group and compare with $\mathrm{BD}$ sera. For further understanding, moesin is a member of the ERM family and highly expressed in cell membranes. It plays a key role in different biological processes, such as cell-cell recognition, leukocyte adhesion and migration, and dynamic interaction with RhoA kinase, VCAM-1 and ICAM-1 $[23,24]$. Moesin can also translocate to the plasma membrane and enhance adhesion of neutrophils on the extracellular surface. Moesin is the major protein of ERM lymphocytes and platelets and differs from ezrin in its insensitivity to calpain [25]. For anti-moesin antibodies, it might participate in the phosphorylation of moesin and increase the migration of neutrophils, which is important in the inflammatory responses [26].

Researchers believe that BD had a close relationship with infection with herpes simplex virus (HSV), which had been considered in disease pathogenesis and its antibodies (anti-HSV) had been identified in serum circulation of BD patients [27]. In our previous study, we also proved that E. coli infection and HSP27 antibody play a part in the process of $\mathrm{BD}[6]$. Moesin regulates actin-plasma membrane cross-linking and microtubule stability. It has been demonstrated that moesin could suppress retroviral infection and HSV by interacting with the cytoskeletal regulatory protein PDZD8 [28]. The presence of anti-moesin antibody may relieve this inhibition effect by accelerating the virus infection.
In conclusion, this study has provided some direct and valuable evidence to show that moesin might be another potential AECA target in patients with $\mathrm{BD}$. With the systematic and reliable screening of antibody profile in patients with BD the difficulty of diagnosis will be greatly reduced and monitoring will hopefully become a thing of the past.

\section{Acknowledgments}

Muhammad Hussain and Peng Chen contributed equally to this work.

This work was supported by the National Natural Science Foundation of China (No. 81571592, 31371203), Beijing Nova Program of Science and Technology (No. 2007B024), Program for New Century Excellent Talents in University (No. NECT12-0773) and the Fundamental Research Funds for the Central public welfare research institutes (ZZ11-113).

\section{Conflict of interest}

The authors declare no conflict of interest.

\section{References}

1. Kurokawa MS, Suzuki N. Behcet's disease. Clin Exp Med 2004; 4: 10-20.

2. Cho SB, Ahn KJ, Kim DH, et al. Identification of HnRNP-A2/B1 as a target antigen of anti-endothelial cell IgA antibody in Behcet's disease. J Invest Dermatol 2012; 132: 601-8.

3. Dinc A, Takafuta T, Jiang D, et al.Anti-endothelial cell antibodies in Behçet's disease.Clin Exp Rheumatol 2003; 21 (4 Suppl 30): S27-30.

4. Aydıntug AO, Tokgöz G, D’Cruz DP, et al. Antibodies to endothelial cells in patients with Behçet's disease. Clin Immunol Immunopathol 1993; 67: 157-62.

5. Chen P, Yan H, Tian Y, et al. Annexin A2 as a target endothelial cell membrane autoantigen in Behcet's disease. Sci Rep 2015; 5: 8162.

6. Chen P, Shi L, Jiang Y, et al. Identification of heat shock protein 27 as a novel autoantigen of Behçet's disease. Biochem Biophys Res Commun 2015; 456: 866-71.

7. Xun Y, Chen P, Yan H, et al. Identification of prohibitin as an antigen in Behcet's disease. Biochem Biophys Res Commun 2014; 451: 389-93.

8. Chen P, Yang W, Tian Y, et al.Electron transfer flavoprotein subunit beta is a candidate endothelial cell autoantigen in behcet's disease. PLoS One 2015; 10: e0124760.

9. Hussain M, Chen P, Mei G, et al. Expression correlates between Annexin $\mathrm{A} 1$ and $\mathrm{A} 2$ autoantibodies in patients with Behcet's Disease. Biosci Rep 2018; 38: BSR20170778.

10. Pearson MA, Reczek D, Bretscher A, et al. Structure of the ERM protein moesin reveals the FERM domain fold masked by an extended actin binding tail domain. Cell 2000; 3: 259-70.

11. Nagao T, Suzuki K, Utsunomiya K, et al. Direct activation of glomerular endothelial cells by anti-moesin activity of anti-myeloperoxidase antibody. Nephrol Dial Transplant 2011; 26: 2752-60. 
12. Suzuki K, Suzuki K, Nagao T, et al. Proposal of anti-moesin as a novel biomarker for ANCA-associated vasculitis. Clin Exp Nephrol 2013; 17: 638-41.

13. Zhao JL, Li MT, Wang Q, et al. Specific anti-moesin antibodies: could it be a novel biomarker of pulmonary involvement associated with systemic sclerosis? Int I Rheum Dis 2010; 13: 29.

14. Takamatsu H, Feng X, Chuhjo T, et al. Specific antibodies to moesin, a membrane-cytoskeleton linker protein, are frequently detected in patients with acquired aplastic anemia. Blood 2007; 109: 2514-20.

15. Hirohata S, Kikuchi H. Behçet's disease. Arthritis Res Ther 2003; 3: 139.

16. Takamatsu H, Espinoza JL, Lu X, et al. Anti-moesin antibodies in the serum of patients with aplastic anemia stimulate peripheral blood mononuclear cells to secrete TNF-alpha and IFN-gamma. I Immunol 2009; 182: 703-10.

17. Weichsler B, Davatchi F, Mizushima Y, et al. Criteria for diagnosis of behcet's disease. Lancet 1990; 335: 1078-80.

18. Du H, Chen G, Wang S, et al. Immunological screening and characterization of highly specific monoclonal antibodies against $20 \mathrm{kDa}$ hGH. Bioanalysis 2012; 4: 2161-8.

19. Wang $X$, Chen P, Cui J, et al. Keratin 8 is a novel autoantigen of rheumatoid arthritis. Biochem Biophys Res Commun 2015; 465: 665-9.

20. Li Y, Wang W, Zhao D, et al. Water-soluble fluorescent CdTe/ZnSe Core/Shell Quantum Dot: aqueous phase synthesis and cytotoxicity assays. J Nanosci Nanotechnol 2015; 15: 4648-52.

21. Miyaji K, Shahrizaila N, Umapathi T, et al. Are ERM (ezrin/radixin/moesin) proteins targets for autoantibodies in demyelinating neuropathies? Hum Immunol 2014; 11: 1089-91.

22. Wagatsuma M, Kimura M, Suzuki R, et al. Ezrin, radixin and moesin are possible autoimmune antigens in rheumatoid arthritis. Mol Immunol 1996; 15: 1171-6.

23. Simoncini T, Scorticati C, Mannella P, et al. Estrogen receptor alpha interacts with Galpha13 to drive actin remodeling and endothelial cell migration via the RhoA Rho kinase/moesin pathway. Mol Endocrinol 2006; 20: 1756-71.

24. Barreiro O, Yáñez-Mó M, Serrador JM, et al. Dynamic interaction of VCAM-1 and ICAM-1 with moesin and ezrin in a novel endothelial docking structure for adherent leukocytes. J Cell Biol 2002; 157: 1233-45.

25. Shcherbina A, Bretscher A, Kenney DM, et al. Moesin, the major ERM protein of lymphocytes and platelets, differs from ezrin in its insensitivity to calpain. FEBS Letters 1999; 443: 31-6.

26. Nakamura F, Amieva MR, Hirota C, et al. Phosphorylation of 558T of moesin detected by site-specific antibodies in RAW264.7 macrophages. Biochem Biophys Res Commun 1996; 226: 650-6.

27. Direskeneli H. Behcet's disease: Infectious aetiology, new autoantigens, and HLA-B51. Ann Rheum Dis 2001; 60: 996-1002.

28. Henning MS, Stiedl P, Barry DS, et al. PDZD8 is a novel moesin-interacting cytoskeletal regulatory protein that suppresses infection by herpes simplex virus type 1 . Virology 2011; 415: 114-21. 